\title{
APLICAÇÃO DE FLOCULANTES EM PÓ PARA SEDIMENTAÇÃO DO RESÍDUO BAYER NA INDÚSTRIA DO ALUMÍNIO
}

\author{
D. A. BORTOLETO ${ }^{*}$, M. A. CAMARgos, J. B DAVO
}

*BASF SA - Mining Solutions, Av. Nações Unidas 14171, São Paulo Brasil. daniel.bortoleto@basf.com**

Submetido 09/10/2017 - Aceito 08/11/2017

DOI: $10.15628 /$ holos.2017.6377

\section{RESUMO}

A produção de alumina através do processo Bayer gera grande quantidade de resíduo conhecido como "lama vermelha". O resíduo é separado do denominado licor verde que contém íons aluminato solubilizados, e direcionado para etapas de separação sólido/líquido realizadas em tanques (lavadores), onde ocorre a dosagem de polímeros solúveis em água que induzem a sedimentação. Utiliza-se sistema de decantação contracorrente com o objetivo de espessar a lama, recuperando o máximo possível de soda cáustica fornecendo um "overflow" contendo baixa concentração de resíduos. O uso de polímeros floculantes em pó, com alto peso molecular e carga iônica adequada, apresenta ganhos significativos relacionados à redução de dosagem, velocidade de sedimentação e compactação do "underflow" resultando em redução significativa de custos no processo produtivo. Entretanto, apesar dos benefícios relatados, muitas refinarias no Brasil ainda fazem uso de polímeros floculantes em emulsão. Nesse trabalho avaliou-se a performance do floculante em pó da Basf (linha Alclar $^{\circledR}$ ) com diferente peso molecular e carga iônica. Avaliou-se também de forma comparativa, a associação de poliacrilamida em pó e na forma de emulsão, ao hidroxamato para avaliar a redução do consumo de hidroxamato nas refinarias, quando se utiliza o floculante em pó da linha $\quad$ Alclar $^{\circledR}$ associado.

PALAVRAS-CHAVE: resíduo Bayer, sedimentação, floculante em pó

\section{POWDER FLOCCULANT APPLICATION FOR BAYER RESIDUE SEDIMENTATION IN THE ALUMINIUM INDUSTRY}

\begin{abstract}
The alumina production through Bayer process generates a large amount of residue known as red mud. Once separated from green liquor (GLQ), red mud is direct to washing steps where synthetic flocculant dosage promotes solid liquid separation. The goal is thickening the slurry to recover caustic sodium as much as possible. The use of powder polyacrylamide with high molecular weight and suitable ionic charge results in significant gains in
\end{abstract}

terms of dosage, settling rate, underflow compaction and overflow turbidity. However, the alumina industry in Brazil still using emulsion flocculants despite the reported benefits. In this paper was evaluated the performance of different powder flocculants from BASF (Alclar ${ }^{\circledR}$ line). In addition, was performed a comparison between powder polyacrylamide and emulsion flocculants associated or not with hydroxamates.

KEYWORDS: Bayer residue; sedimentation; powder flocculant 


\section{INTRODUÇÃO}

A solubilização da alumina contida na bauxita utilizando-se hidróxido de sódio a altas pressões e temperaturas é conhecida como processo Bayer. Este processo resulta na geração de grande quantidade de resíduo de bauxita denominado "lama vermelha". Com base na produção global de alumina em 110 milhões de toneladas e no teor de alumina disponível na bauxita entre 36 e 53\%, estima-se a geração em torno de 150 milhões de toneladas de resíduo anualmente (KILDEA, 2010). Segundo Góis (2003), a "lama vermelha" apresenta como principais componentes hematita, quartzo, goethita, sodalita e granulometria abaixo de $300 \mu \mathrm{m}$.

A etapa de clarificação é muito importante no processo de produção da alumina que consiste na separação sólido/líquido, onde o resíduo de bauxita é separado do licor que contém alumina solubilizada. Até meados da década de 70, ainda se utilizavam floculantes naturais nestas operações, como amido ou farelo de trigo. Aos poucos, a indústria do alumínio passou a utilizar floculantes sintéticos, as poliacrilamidas, de alto peso molecular e carga iônica, devido à baixa performance dos floculantes naturais (PEARSE; SARTOWSKI, 1984). Na década de 80, diante das necessidades do setor do alumínio de obtenção de um "overflow" mais clarificado após a sedimentação da lama, floculantes do tipo hidroxamato foram desenvolvidos baseados em componentes etanoaminas que possuem como característica fundamental, a capacidade de complexar o ferro em sistemas de sedimentação com alta concentração de sólidos, partículas finas e alto teor alcalino (AVOTINS; RYLES, 1996). Normalmente utilizado nos estágios iniciais de sedimentação da "lama vermelha" nas refinarias de alumínio, o hidroxamato pode ser associado à poliacrilamida para otimização da performance do processo.

A acrilamida é produzida a partir da hidrólise da acrilonitrila que, por sua vez é sintetizada a partir do propileno. Neste processo, propileno, amônia e ar passam por reator de leito fluidizado contendo um catalizador. Uma vez sintetizada, a acrilamida é polimerizada formando a poliacrilamida. O processo catalítico ocorre através da quebra da dupla ligação entre os carbonos 2 e 3 do monômero, produzindo radicais livres produzindo uma reação em cadeia entre os monômeros (KIM; HYUN, 2002).

A floculação da hematita mineral, comum no resíduo Bayer em meios altamente alcalinos ocorre através do mecanismo de pontes não simétricas onde o polímero realiza o ataque a uma ou mais partículas gerando moléculas de cadeia longa que passam a interagir entre si através de pontes de hidrogênio, força de Van der Waals e interações iônicas. Desta forma, o aumento do tamanho das partículas resulta na maior eficiência da separação sólido/líquido (PATNAIK, 2000). Segundo Senaputra (2012), a etapa de dessilicação é fundamental para otimização da performance da separação sólido/líquido da "lama vermelha" devido ao aumento da área superficial disponível dos floculantes.

Apesar da melhor performance da poliacrilamida em pó, como será demonstrado nos resultados a seguir, a indústria do Alumínio no Brasil tradicionalmente utiliza a poliacrilamida na forma de emulsão. Com base nos resultados experimentais em bancada, testes industriais e aplicação regular, floculantes em pó (linha Alclar $\left.^{\circledR}\right)$ possuem custo até $70 \%$ menor para a refinaria comparado a floculantes similares na forma de emulsão, além de apresentar maior velocidade de sedimentação, maior compactação do underflow e menor turbidez do overflow. 
Nesse trabalho foi realizado um estudo da sedimentação da "lama vermelha" proveniente do Processo Bayer de uma refinaria de alumínio, utilizando-se floculante em pó da BASF (linha Alclar ${ }^{\circledR}$ ), a base de poliacrilamida de cadeia longa e alta carga iônica. Também se realizou um estudo comparativo entre o floculante BASF em pó, Alclar ${ }^{\circledR} 3000$, com floculante similar na forma de emulsão produzido por outro fabricante. Avaliou-se também de forma comparativa, a associação de poliacrilamida em pó e na forma de emulsão, ao hidroxamato. O motivo foi avaliar a redução do consumo de hidroxamato nas refinarias, produto de alto valor agregado, quando se utiliza o floculante em pó da linha Alclar ${ }^{\circledR}$.

\section{METODOLOGIA}

A primeira etapa do trabalho consistiu na preparação da solução aquosa de floculante. Pesou-se em balança analítica $0,1 \mathrm{~g}$ do Alclar $^{\circledR}$ e $500 \mathrm{~g}$ de água que foram submetidos à agitação manual, para obtenção de uma solução com concentração igual a $0,02 \%$ para os ensaios laboratoriais. Em seguida, uma amostra representativa da "lama vermelha" com concentração igual a $33 \mathrm{~g} / \mathrm{l}$ foi selecionada a partir do processo Bayer que, uma vez devidamente homogeneizada, foi transferida para provetas graduadas de $1000 \mathrm{ml}$ e colocadas em recipiente de banho térmico em torno de 95oC. O material deve ser homogeneizado novamente, desta vez dentro das provetas com o uso de agitador tipo "plunger", antes do início de cada ensaio de sedimentação. O floculantes foram adicionados às provetas com auxílio de pipeta, nas dosagens adequadas ao estudo, entre 15 e 35 gramas de floculante por tonelada de lama. Nova agitação foi realizada com auxílio do "plunger", desta vez em movimentos lentos repetindo-se 4 vezes. Imediatamente após esta etapa, o tempo de sedimentação foi medido entre os volumes 900 e $700 \mathrm{ml}$ das provetas para estimativa das velocidades, expressas em metros por hora $(\mathrm{m} / \mathrm{h})$. A leitura da compactação da "lama vermelha" assim como a turbidez do sobrenadante foram realizadas após 10 minutos. A compactação e a turbidez foram expressas em volume (ml) e NTU respectivamente. Desta forma, através desta metodologia avaliou-se diferentes floculantes em pó da Basf (Alclar HP21, HP22, 662 e 3000), assim como ensaios comparativos com floculantes em emulsão, associados ou não ao hidroxamato.

\section{RESULTADOS E DISCUSSÃO}

$\mathrm{Na}$ Figura 1, estão apresentados os resultados da velocidade de sedimentação de diferentes floculantes da linha Alclar $^{\circledR}$ utilizando-se a mesma amostra de "lama vermelha". A diferença entre os floculantes está no tamanho das cadeias da poliacrilamida, assim como na carga iônica das moléculas. 


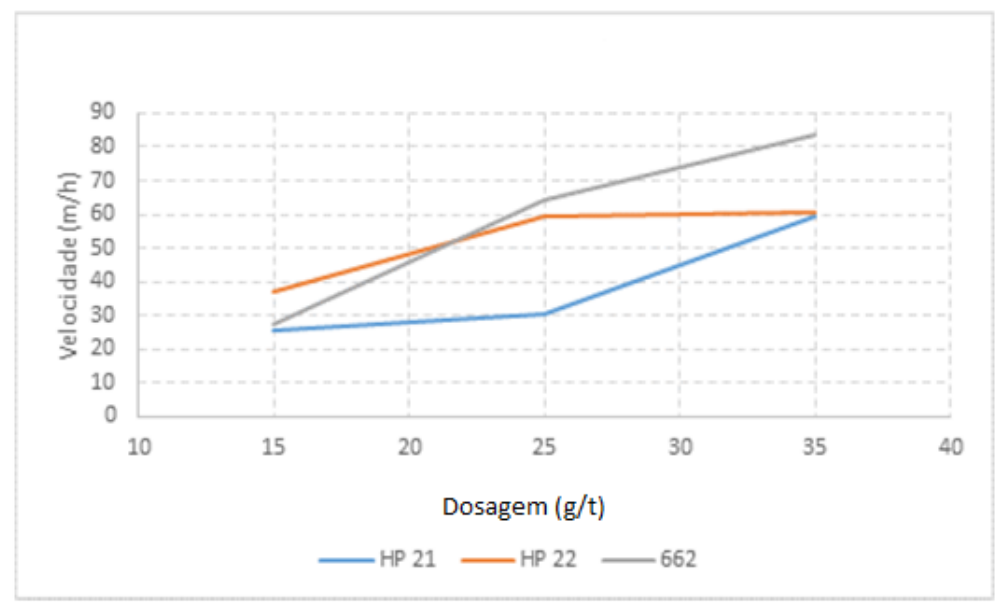

Figura 1: Velocidade de sedimentação da lama vermelha

Existem fatores que exercem influência na performance dos floculantes durante a sedimentação da "lama vermelha" como, a especificação da bauxita, concentração de sólidos dos lavadores, teor alcalino, teor de hematita, goethita, granulometria, entre outros. Desta forma, a variação no peso molecular e carga iônica das moléculas de poliacrilamida, permite maior abrangência sobre a variabilidade do processo de produção da refinaria de alumínio.

Na Figura 2 estão os resultados de turbidez em NTU do overflow da "lama vermelha" para o mesmo experimento. Observa-se que os floculantes que apresentaram maior velocidade de sedimentação, obtiveram maior turbidez no overflow. O motivo é que em maiores velocidades, não há tempo hábil de captura das partículas mais finas, fato que deve ser ponderado na seleção do floculante ideal para a realidade de cada etapa do processo.

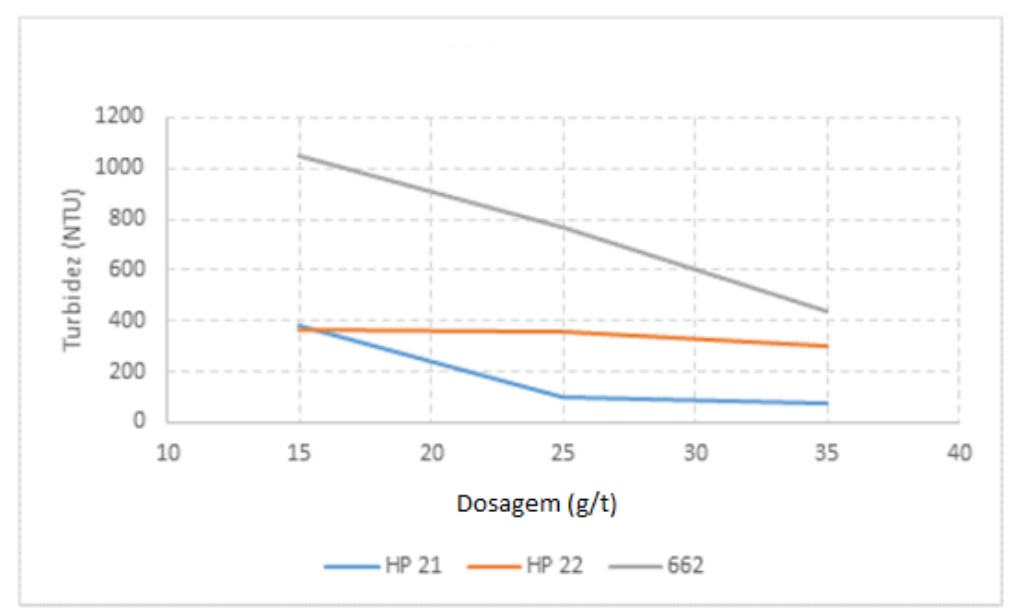

Figura 2: Turbidez do overflow (NTU) da lama para diferentes floculantes da linha Alclar

Na Figura 3 estão apresentados os resultados de compactação do underflow da "lama vermelha". Este parâmetro é muito importante, visto que um dos principais objetivos da sedimentação nos lavadores é a recuperação de soda cáustica para o processo produtivo. Assim, quanto maior a compactação do underflow, menor quantidade de soda está sendo enviada para o rejeito. 


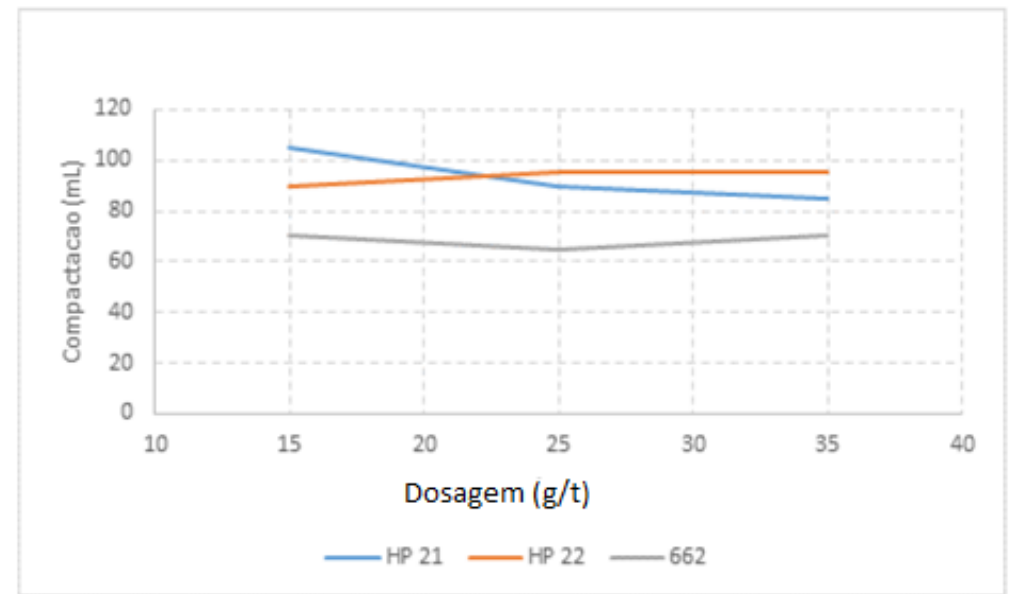

Figura 3: Compactação do underflow $(\mathrm{ml})$ da lama para diferentes floculantes da linha Alclar

Nas próximas figuras estão os resultados comparativos obtidos em laboratório, entre poliacrilamida em pó (linha Alclar ${ }^{\circledR}$ ) da Basf e similar de outro fabricante na forma de emulsão. No caso específico deste processo avaliado, devido às características granulométricas da bauxita utilizada, o estágio inicial de lavagem exige o uso de hidroxamato. Neste caso, se utilizou o Alclar 3000 e a emulsão, associados ao hidroxamato 1:1 nesta etapa (Figura 4).

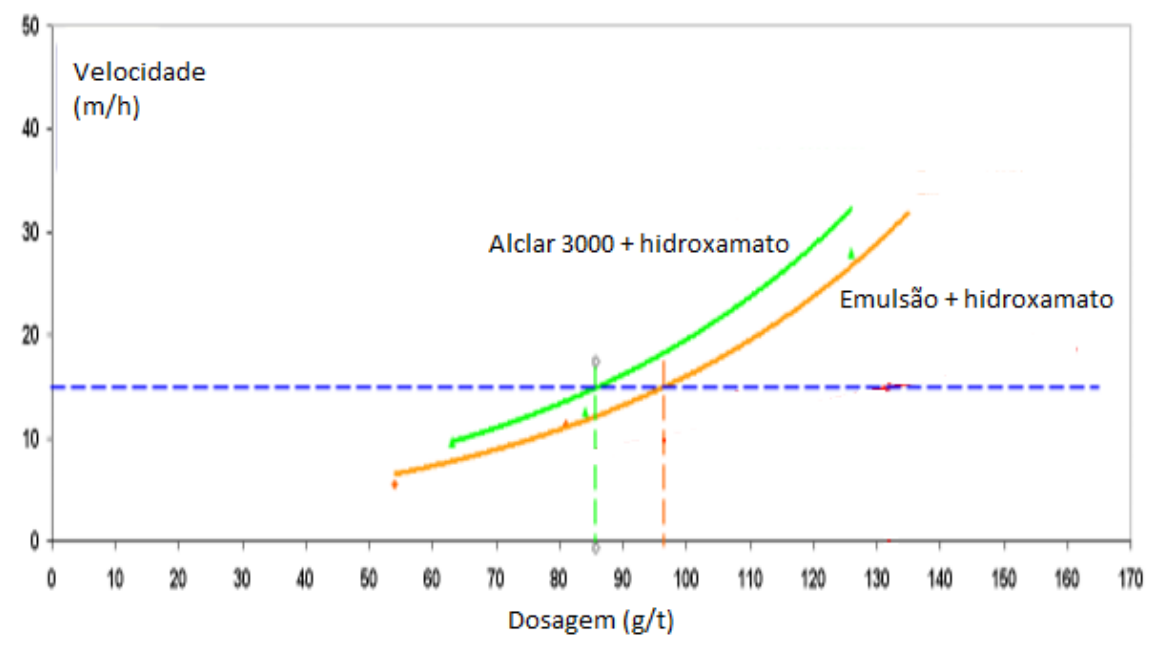

Figura 4. Velocidade de sedimentação no estágio inicial do processo

Observa-se que a velocidade de sedimentação da lama sob a ação do Alclar $^{\circledR} 3000$, quando associado ao hidroxamato é maior que, quando o hidroxamato está associado à emulsão. A principal consequência é o menor consumo, tanto da poliacrilamida quanto do hidroxamato gerando redução de custos para a refinaria.

Na Figura 5 estão apresentadas as velocidades de sedimentação no estágio intermediário de lavadores do processo avaliado. 


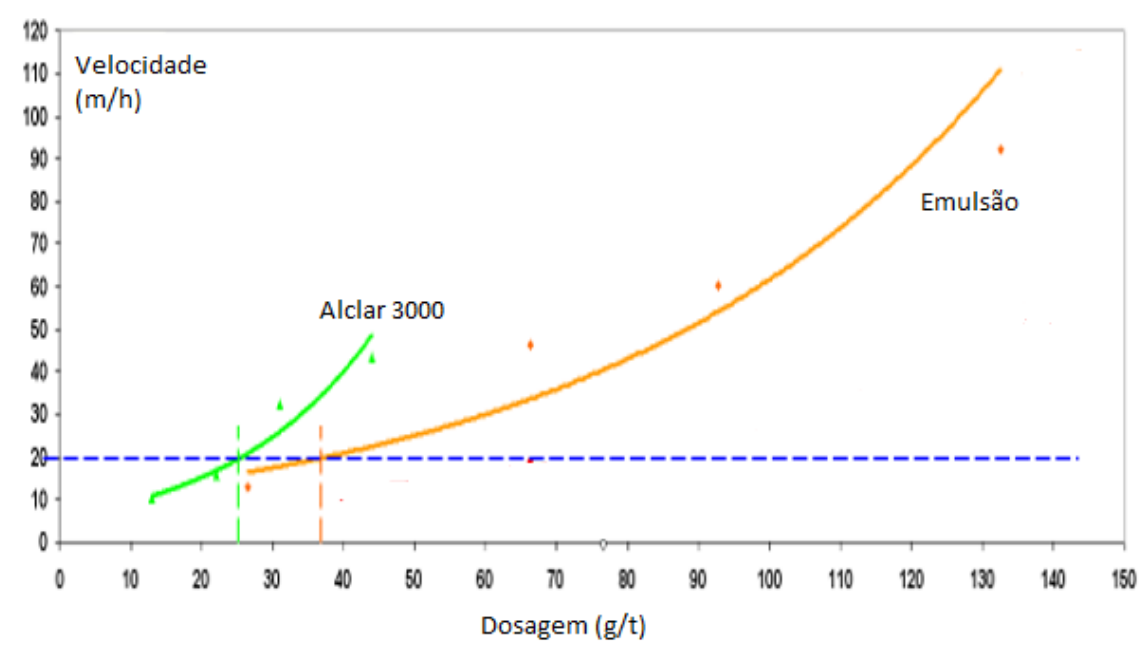

Figura 5. Velocidade de sedimentação no estágio intermediário do processo

No estágio intermediário, quando se utiliza no processo somente a poliacrilamida, a redução da dosagem é considerável com o uso do Alclar ${ }^{\circledR}$ 3000. Da mesma forma ocorre no estágio final de lavadores do processo avaliado (Figura 6).

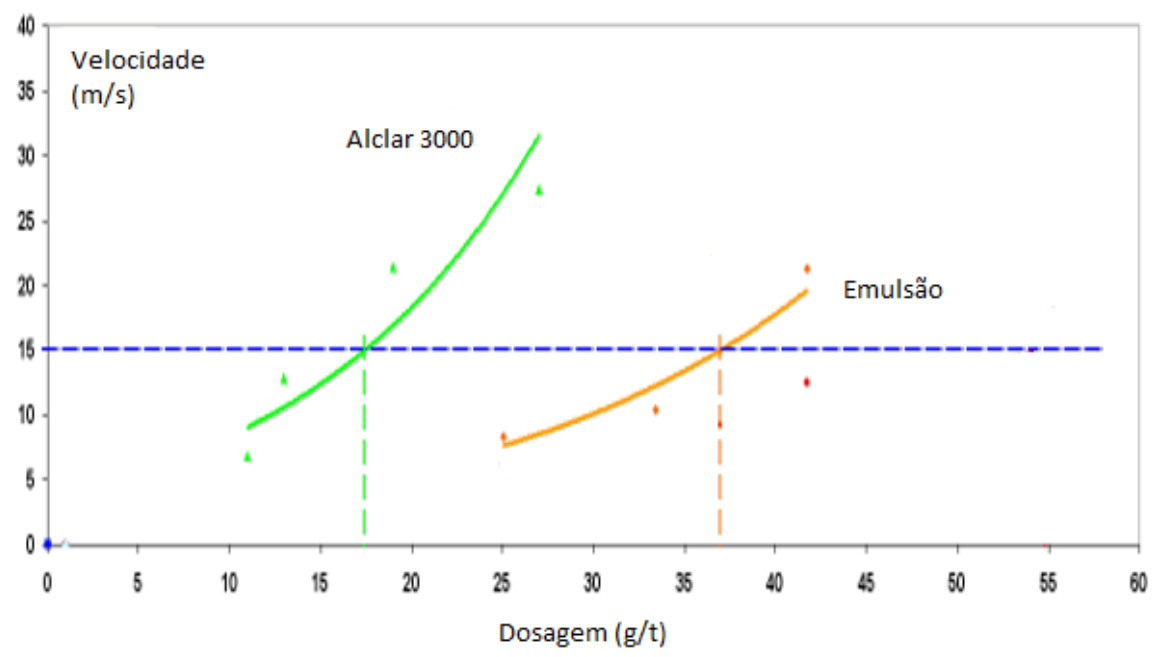

Figura 6. Velocidade de sedimentação no estágio final do processo

No estágio final do processo avaliado, o consumo de poliacrilamida é em torno de três vezes menor quando se utiliza o Alclar $^{\circledR} 3000$.

\section{CONCLUSÕES}

O uso da poliacrilamida na forma de emulsão, vem sendo substituído pela forma de pó em várias refinarias no mundo para sedimentação do resíduo Bayer. O motivo é a menor dosagem, maior velocidade de sedimentação, maior compactação do "underflow" e menor turbidez do "overflow" comprovado com anos de uso regular. Os estudos de sedimentação realizados no presente trabalho, além de comprovarem estes resultados, comprovam a possibilidade de redução do consumo de hidroxamato, que possui alto valor agregado, nos primeiros estágios de sedimentação da "lama vermelha" nas refinarias que utilizam este produto. Isto ocorre devido ao maior sinergismo do hidroxamato na forma de pó (linha Alclar ${ }^{\circledR}$ ), do que com a poliacrilamida na forma de emulsão. 
Ensaios de sedimentação devem ser realizados a fim de se identificar a poliacrilamida com peso molecular e carga iônica ideais para cada processo, pois existem diversas variáveis, desde a qualidade da bauxita até a concentração de sólidos e teor alcalino da "lama vermelha".

\section{REFERÊNCIAS}

AVOTINS, P.V., RYLES R. G. Superfloc H.X, a new technology for alumina industry. Inc. p. 1-11, 1996.

GÓIS, C.G., LIMA, R.M.F. (2003). Sedimentação de resíduo Bayer utilizando floculantes hidroxamato e poliacrilamida. REM: R.Esc. Minas Ouro Preto. P.119-122.

KILDEA, J. (2010). New developments in red mud flocculation and control of solid/liquid separation, P. 1-8.

KIM, B., HYUN, H.H. (2002). Biotechnology and Bioprocess Engineering

PATNAIK, S.K., MURTHY, P.V.R. (2000). Importance of synthetic flocculants in alumina industry. Processing of Fines. P. 249-254

PEARSE, M.J., SARTOWSKI, Z. (1984). Aplications of special chemicals (flocculants and dewatering aids) for red mud separation and hydrate filtration. Bauxite Society of Mining Engineers. P. 775-787

SENAPUTRA, A., FAWELL, P. (2012).The impact of desilication product on bauxite residue flocculation. Proceedings of the 9th International Alumina Quality Workshop. P. 186-192. 